Case Report

\title{
New onset of fever and lymphadenopathy in a patient on antitubercular treatment for tuberculous meningitis:
}

\section{Drug resistance or paradoxical response phenomenon?}

\author{
D Majumdar ${ }^{1}$, S Chakraborty ${ }^{1}$, A Naskar, ${ }^{1}$ MK Ghosh ${ }^{1}$, \\ B Saha ${ }^{1}$ \\ Sri Lankan Journal of Infectious Diseases 2017 Vol.7 (1):58-62 \\ DOI: http://doi.org/10.4038/sljid.v7i1.8123
}

\begin{abstract}
Central nervous system (CNS) involvement is one of the most devastating clinical manifestations of tuberculosis (TB) noted in 5 to $10 \%$ of patients with extra pulmonary TB and accounts for approximately $1 \%$ of all patients with TB. Clinical deterioration during treatment with anti-tuberculosis drugs (ATD) is not uncommon in immunocompetent persons. Possibilities include multi-drug resistant tuberculosis (MDR-TB), a paradoxical response or some other non-tubercular causes of fever. Corticosteroids are considered to have a beneficial effect in the management of paradoxical reactions. Immuno-modulatory drugs, including tumour necrosis factor- $\alpha$ antagonists, thalidomide and interferon- $\gamma$ have been used in isolated cases with more severe forms of paradoxical reactions. We report here a case of a 13 year old girl who while being treated for tubercular meningitis, developed high grade fever with lymphadenopathy. Her lymph node biopsy showed caseous necrosis with presence of AFB suggestive of a paradoxical reaction.
\end{abstract}

Keywords: Paradoxical response, Immunocompetent, Tuberculosis, MDR TB

\section{Introduction}

Tuberculosis (TB) can evolve to severe forms and advanced disease requiring mechanical ventilation of the patients. Worsening condition of TB under therapy should be carefully handled by health practitioners. This condition develops due to several reasons such as poor compliance, malnutrition, poor drug absorption, slow response to ATD therapy associated with immunocompromised status and MDR-TB. A paradoxical response has to be considered as the cause after all other possible causes have been excluded. The paradoxical response phenomenon is defined as the clinical or radiological worsening during initiation of ATD therapy, related to temporary recovery of the immune system. ${ }^{1}$ A paradoxical response has been reported to occur in 5-35\% of cases, mostly in immunocompromised patients such as those with HIV. ${ }^{2,3}$ The paradoxical phenomenon generally occurs ranging from 20 to 109 days in HIV negative patients and predominantly involves the central nervous system (CNS), respiratory system and lymphoreticular system. It poses serious problems in the management of CNS tuberculosis. There is always a concern about development of drug resistant

${ }^{1}$ Dept of Tropical Medicine, School of Tropical Medicine, Kolkata, India

Address for correspondence: Dr. Debajyoti Majumdar Department of Tropical Medicine, School of Tropical Medicine, 108, Chittaranjan Avenue, Kolkata, West Bengal, India, Pin 700073 Telephone: +919804330792 ; Email: dr.debajyotimajumdar@gmail.com 
tuberculosis when there is clinical deterioration of the patient which often leads to inappropriate increment or addition of more toxic newer antitubercular drugs with adverse consequences.

\section{Case report}

We report a 13 year old girl who presented with fever for 1 month and headache with blurring of vision. She had a positive history of contact with her elder sister suffering from sputum positive pulmonary TB, who was already receiving Category I anti tubercular therapy from a local Directly Observed Treatment, Short Course (DOTS) centre. None of the family members were vaccinated with BCG.

The patient was conscious, cooperative but drowsy on admission. Clinical examination revealed mild hepatomegaly without any abnormal chest findings or peripheral lymphadenopathy. Neck rigidity was present. There was no sign of any focal neurological deficit. Funduscopy was normal. Routine blood investigations along with cerebrospinal fluid (CSF) study and magnetic resonance imaging (MRI) of the brain with contrast was done. All the surveillance cultures were sent to look for any foci of bacterial infection. Ultrasonography of the whole abdomen and chest X-ray were done which didn't reveal any abnormality.

The patient was started on empirical intravenous (IV) ceftriaxone on day 1 on a presumptive clinical diagnosis of bacterial meningitis. Blood investigations were within normal limits and cultures didn't show any growth. She was nonreactive for HIV I and II, HBsAg, Anti HCV and VDRL. CSF showed high protein $(107 \mathrm{mg} / \mathrm{dl})$ and low glucose of $36 \mathrm{mg} / \mathrm{dl}$ (corresponding blood glucose $96 \mathrm{mg} / \mathrm{dl}$ ) with lymphocytic pleocytosis $\left(85 \mathrm{cells} / \mathrm{mm}^{3}\right.$, all lymphocytes). CSF adenosine deaminase (ADA) was low (3.5 u/l) and culture showed no growth. Fungal stain and culture were negative.

The patient was started on antitubercular therapy with isoniazid, rifampicin, ethambutol and pyrazinamide along with IV dexamethasone (according to weight) on day 2 after admission and ceftriaxone was discontinued. Her fever decreased both in amplitude and frequency after 6 days of treatment and sensorium improved. However, she developed a high spike of fever with appearance of headache and diplopia on day 10. All routine investigations along with surveillance cultures were repeated. Lumbar puncture was carried out after fundoscopic examination. The CSF protein was increased $(191.7 \mathrm{mg} / \mathrm{dl})$ and glucose decreased $(6 \mathrm{mg} / \mathrm{dl})$ from previous values. Rifampicin sensitive Mycobacterium tuberculosis was detected in the CSF by Cartridge Based Nucleic Acid Amplification Test (CBNAAT). All other investigations were within normal limits. Keeping the possibility of pyogenic meningitis in mind as the CSF glucose was very low, IV meropenem (as she already received $3^{\text {rd }}$ generation cephalosporin before starting ATD) along with vancomycin (for penicillin resistant pneumococci) were started to which there was no clinical response. After 5 days she developed right cervical lymphadenopathy $\left(1.5 \times 1.0 \mathrm{~cm}^{2}\right)$ and a few small axillary lymph nodes. Fine needle aspiration (FNA) from the lymph node showed only bloody elements and FNA sample for CBNAAT didn't reveal the presence of $M$. tuberculosis. Lymph node biopsy was done which showed presence of caseous necrosis with acid fast bacilli (AFB).

We now considered the possibility of drug resistant TB (to drugs other than rifampicin) or a paradoxical response. With those two differentials in mind, streptomycin and levofloxacin were added (as per Revised National Tuberculosis Control Program guidelines, suspecting isoniazid resistance) to strengthen the ATD regimen as the patient couldn't afford drug 
sensitivity testing. In spite of this six drugs combination for another ten days, her fever did not respond. Prednisolone was then added to cover a possible paradoxical response.

The patient became afebrile with regression of lymph nodes 3 days after onset of steroid therapy. She was discharged from the hospital 72 hours after she became afebrile with advice to continue the six drug anti tubercular regimen for 3 months followed by isoniazid and rifampicin for the next 8 months. Her steroid regime was tapered over the next 4 weeks. On follow up visits over the next six months she remained afebrile, healthy and showed an adequate weight gain.

\section{Discussion}

Severe manifestations during TB therapy occur occasionally in immunocompetent adolescents. Cruz et al (2013) report the presentation of adolescents aged under 18 years presenting to emergency departments most commonly with seizure and altered mental status. ${ }^{4}$ Severe respiratory distress and altered mental status could be other major causes of patient admission to intensive care and contributing to poor prognosis. ${ }^{1,5}$

Deterioration of clinical manifestations in patients with TB during treatment may be due to many causes including secondary infections, inadequate ATD therapy, poor compliance, poor drug absorption, MDR-TB and the paradoxical response. ${ }^{1,2}$

A broad spectrum cephalosporin was given to this patient for possible secondary infection. She was under directly observed treatment and there was no history suggestive of malabsorption syndrome. CBNAAT examination on CSF was positive for $M$. tuberculosis sensitive to rifampicin (sensitivity and specificity $95.7 \%$ and $99.3 \%$ respectively $^{6}$ ).

Repeated culture and smear negative results in a patient with clinical and radiographical deterioration may indicate that the patient has causes other than MDR-TB. ${ }^{7}$ Paradoxical response should be considered as a differential diagnosis of MDR-TB, if other causes had been excluded. Paradoxical response predominantly happens in extrapulmonary and disseminated TB, like miliary TB and TB meningitis. ${ }^{8}$ Median regression time of paradoxical response is 46 days, ranging from 14 to 120 days with a mean of 63 days for CNS involvement and 56 days for other site involvement. ${ }^{8}$ Other factors that strengthened the possibility of a paradoxical response was lack of evidence of BCG vaccination. Olive, et al $(2013)^{8}$ found children who suffered paradoxical response had not received the BCG vaccine, which is thought to offer protection against this complication.

There is no specific treatment for the paradoxical response. Optimal and close evaluation is needed. Continuation of the same anti-TB treatment and if relevant, initiation of corticosteroid therapy are the mainstay of management. The total duration of ATD treatment is not influenced by the development of paradoxical response. ${ }^{8,9,10}$ In this patient, because symptoms and signs of MTB infection were present in spite of a six drugs regimen, oral corticosteroid was added while continuing ATD as it is effective in regulation of the immune system. $^{2}$

Paradoxical response is now increasingly being recognized as a cause of subsequent deterioration in cases of CNS tuberculosis and some extrapulmonary TB despite adequate and appropriate therapy. This phenomenon complicates the decision about the therapy of CNS tuberculosis and other extrapulmonary TB. It is not possible to clearly differentiate between 
paradoxical deterioration and development of secondary resistance in the absence of positive tests of culture and sensitivity for $M$. tuberculosis. CSF culture is positive for M. tuberculosis in less than $50 \%$ of clinically diagnosed cases of TB meningitis.

The most likely explanation for paradoxical response is aninterplay between the host's immune response and the direct effect of mycobacterial products. Active tuberculosis leads to depression of the delayed type of hypersensitivity response due to activation of monocytes by protein derivatives of mycobacteria. Specific antigens such as D-arabinose-D-galactose from the tubercle bacilli lead to the production of immunosuppressive concentration of prostaglandin-E2. Once active tuberculosis is under control and immunosuppression is resolved, enhanced delayed type hypersensitivity and, activation and accumulation of lymphocytes and macrophages at the site of bacterial deposition or toxin production occurs when bacilli die. The reason for occurrence of this response in only some cases and not all suggests that it depends on host immune responses, virulence of tubercle bacilli, antigen load and effective antitubercular therapy. Corticosteroids and nonsteroidal anti-inflammatory drugs have been used to treat immune reconstitution inflammatory syndrome (IRIS). A double blind, randomized, placebo-controlled clinical trial for corticosteroids was performed in patients with paradoxical TB-IRIS. Prednisone significantly reduced days of hospitalization and outpatient therapeutic procedures, more rapidly improving symptoms, quality-of life score and chest radiography. ${ }^{11}$

In view of AIDS and MDR tuberculosis, it is essential that particular attention be paid to isolation of $M$. tuberculosis and determination of its sensitivity at the outset. Review of the literature suggests that centrifuging CSF, preparing thick smears from cob-web coagulum and increasing the examination time can increase the yield of AFB from CSF. Repeated lumbar puncture and CSF examination can increase the yield to $87 \%{ }^{12}$. However, CSF smear positive for tubercle bacilli may not necessarily be positive on culture. In the final analysis, clinical judgement, regular follow up, guarded reassurance of patient and recognition of the possibility of paradoxical response is the only practical answer.

\section{Conclusion}

A patient with tuberculosis who deteriorates on treatment sometimes provokes unnecessary changes in the antitubercular regimen along with other supportive measurement especially where culture is negative or not available. Paradoxical worsening in this scenario should be strongly considered. Recognition of this phenomenon will avoid unnecessary addition of antitubercular drugs and improve the morbidity and mortality. The solution to this dilemma of paradoxical response with negative laboratory support, lies in close monitoring of patient with continuation of drugs already in use with addition of steroid, increasing the dose of drugs already in use and/or addition of second line ATT

\section{Conflict of Interest: None}

\section{References}

1. Cheng VCC, Ho PL, Lee RA, et al. Clinical spectrum of paradoxical deterioration during antituberculosis therapy in non HIV infected patients. Eur J Clin Microbiol Infect Dis. 2002; 21:803-9 doi : http://dx.doi.org/10.1007/s10096-002-0821-2

2. Park JA, Park SS, Park SE. A paradoxical reaction during antituberculosis therapy for congenital tuberculosis. Int J Infect Dis. 2009; 13:279-81. 
doi : http://dx.doi.org/10.1016/j.ijid.2008.11.005

3. Cheng VCC, Yam WC, Woo PCY, et al. Risk factors for development of paradoxical response during antituberculosis therapy in HIV-negative patients. Eur J Clin Microbiol Infect Dis. 2003; 22:597-602 doi : http://dx.doi.org/10.1007/s10096-0030998-z

4. Cruz AT, Hwang KM, Birnbaum GD, et al. Adolescents with tuberculosis A review of 145 cases. Pediatr Infect Dis J 2013; 32:937-41

doi : http://dx.doi.org/10.1097/INF.0b013e3182933214

5. Valade S, Raskine L, Aout M, et al. Tuberculosis in the intensive care unit: A retrospective descriptive cohort study with determination of a predictive fatality score. Can J Infect Dis Med Microbiol 2012; 23(4):173-78 No doi

PMCID: PMC3597393

6. Surendra K Sharma, Mikashmi Kohli, Raj Narayan Yadav, et al. Evaluating the diagnostic accuracy of Xpert MTB/RIF assay in pulmonary tuberculosis, PLoS One. 2015; 10(10): e0141011. doi: 10.1371/journal.pone.0141011

7. Perez-Velez CM, Marais BJ. Current concepts tuberculosis in children. $N$ Engl $J$ Med. 2012; 367:348-61. doi : http://dx.doi.org/10.1056/NEJMra1008049

8. Olive $\mathrm{C}$, Mouchet $\mathrm{F}$, Toppet $\mathrm{V}$, et al. Paradoxical reaction during tuberculosis treatment in immunocompetent children: Clinical spectrum and risk factors. Pediatr Infect Dis J. 2013; 32:446-49.

doi : http://dx.doi.org/10.1097/INF.0b013e3182846c4e

9. Hawkey CR, Yap T, Pereira J, et al. Characterization and management of paradoxical upgrading reactions in HIV-uninfected patients with lymph node tuberculosis. Clin Infect Dis. 2005; 40:1368-71. doi : http://dx.doi.org/10.1086/429317

10. Ali U, Chikar S, Patil M, et al. Acute respiratory distress during paradoxical reaction to antitberculosis therapy in an 8 month old child. Lung India. 2012; 29:381-3.

11. Lanzafame M., Vento S. Tuberculosis-immune reconstitution inflammatory syndrome, Journal of Clinical Tuberculosis and Other Mycobacterial Diseases 2016; 3:6-9 doi : http://doi.org/10.1016/j.jctube.2016.03.002

12. Meena Gupta, BK Bajaj, Geeta Khwaja, Paradoxical response in patients with CNS tuberculosis Journal of The Association of Physicians of India 2003; 51:257-263 No doi 